\title{
GATA binding protein 2 overexpression is associated with poor prognosis in KRAS mutant colorectal cancer
}

\author{
KAI XU ${ }^{1 *}$, JIAYUAN WANG $^{2 *}$, JING GAO $^{2}$, JIABO DI $^{1}$, BEIHAI JIANG ${ }^{1}$, LEI CHEN $^{1}$, ZAOZAO WANG $^{1}$, \\ AIDONG WANG $^{1}$, FAN WU $^{1}$, WEI WU ${ }^{1}$, LIN SHEN $^{2}$ and XIANGQIAN SU ${ }^{1}$ \\ ${ }^{1}$ Key Laboratory of Carcinogenesis and Translational Research (Ministry of Education), Department of \\ Gastrointestinal Surgery IV, Peking University Cancer Hospital and Institute; ${ }^{2}$ Key Laboratory of Carcinogenesis \\ and Translational Research (Ministry of Education), Department of Gastrointestinal Oncology, \\ Peking University Cancer Hospital and Institute, Beijing 100142, P.R. China
}

Received February 6, 2016; Accepted June 21, 2016

DOI: 10.3892/or.2016.4961

\begin{abstract}
Colorectal cancer (CRC) is one of the most lethal cancers worldwide. Mutations in KRAS occur with the frequency of $30-50 \%$ in CRC leading to decreased therapeutic response to anti-epidermal growth factor receptor (EGFR) agents. Recently GATA2 was proven to be essential in the survival of KRAS mutant non-small cell lung cancer (NSCLC) cells. However, the association between KRAS mutation and GATA2 expression in CRC remains largely unknown. In the present study, dideoxy sequencing and immunohistochemistry were used to determine KRAS mutation and GATA2 expression, respectively, in a cohort of 236 patients. Cox proportional hazard regression and Kaplan-Meier survival analysis were performed to study the association between KRAS mutation or GATA2 expression and clinical outcomes. Kaplan-Meier
\end{abstract}

Correspondence to: Professor Lin Shen, Key Laboratory of Carcinogenesis and Translational Research (Ministry of Education), Department of Gastrointestinal Oncology, Peking University Cancer Hospital and Institute, 52 Fucheng Road, Haidian, Beijing 100142, P.R. China

E-mail:1in100@medmail.com.cn

Professor Xiangqian Su, Key Laboratory of Carcinogenesis and Translational Research (Ministry of Education), Department of Gastrointestinal Surgery IV, Peking University Cancer Hospital and Institute, 52 Fucheng Road, Haidian, Beijing 100142, P.R. China

E-mail: suxiangqian@bjmu.edu.cn

*Contributed equally

Abbreviations: CRC, colorectal cancer; GATA2, GATA2-binding protein 2; EGFR, epidermal growth factor receptor; NSCLC, non-small cell lung cancer; DFS, disease-free survival; PTEN, phosphatase and tensin homologue; FFPE, formalin-fixed, paraffinembedded; PCR, polymerase chain reaction

Key words: colorectal cancer, KRAS mutation, GATA2, prognosis, survival analysis revealed that KRAS mutant patients with high expression of GATA2 had significantly worse long-term clinical outcomes than those with low expression of GATA2 $(\mathrm{P}<0.001)$. Further analysis showed that patients with both KRAS mutation and high GATA2 expression experienced significantly more unfavorable 5-year outcomes than patients with wildtype KRAS and low GATA2 expression $(\mathrm{P}=0.001)$. Univariate and multivariate Cox proportional hazard regression demonstrated the GATA2 expression level was an independent risk factor for overall survival of CRC patients (HR 1.645; 95\% CI 1.004-2.696; $\mathrm{P}=0.048$ ). In conclusion, the results of this study demonstrated that high expression of GATA2 is correlated with worse survival outcomes in KRAS mutant CRC patients, suggesting that GATA2 may serve as a novel biomarker for the survival of CRC patients harboring KRAS mutation.

\section{Introduction}

Colorectal cancer (CRC) is one of the most prevalent cancers worldwide and the third ranked fatal malignancy in the United States $(1,2)$. Although there has been progress in diagnosis, surgery, combined chemotherapy and targeted therapy $(3,4)$, CRC remains a significant adverse influence on human health with the 5-year survival of only $65 \%$ (5). KRAS has long been known as the most frequently mutated gene in nearly all types of cancers. Particularly, approximately $40 \%$ of the CRCs harbor a mutation in KRAS (6). Mutations in codons 12 and 13 in exon 2 accounts for $90 \%$ of all KRAS mutations, and KRAS gene mutation test has been used as a predictor clinically to determine the lack of efficacy on targeting agents such as cetuximab and panitumumab (7-10). Although still controversial, several studies have demonstrated that KRAS mutation in colorectal carcinoma is correlated to unfavorable survival and enhanced tumor aggressiveness $(11,12)$. However, recent therapeutic targeting of RAS in CRC has proven intractable, due to frequent mutation in KRAS (13). Therefore, searching for new therapeutic targets within the RAS signaling network is necessary and strongly warranted.

GATA binding protein 2 (GATA2), a key member of zinc finger transcription factors family, is identified as a critical regulator of growth, differentiation and survival of 
hematopoietic stem cells (14-16). Increasing evidence has shown that GATA2 expression is correlated with hematologic pathophysiologies and the proliferation and progression of solid tumors (17-23). It was reported that overexpression of GATA2 would contribute to the development of breast cancer through negatively regulating the transcription of phosphatase and tensin homologue (PTEN) (20). As for prostate cancer, upregulated GATA2 expression has been reported to be correlated with tumor progression and GATA2 has been suggested to be a pioneer factor in the regulation of androgen receptor related genes $(21,22)$.

Kumar et al demonstrated that RAS-pathway mutant nonsmall cell lung cancer (NSCLC) cells depended on GATA2 for viability and regulation of the GATA2-related signaling pathways remarkably provoking regression of NSCLC (24). Further studies proved that delivery of GATA2 siRNA with selected carrier downregulated GATA2 expression and caused pronounced synthetic lethal effect of NSCLC in vivo (25). However, the association between KRAS mutation and GATA2 expression and its prognostic value in CRC remains unexplored.

In this study, we performed immunohistochemistry to examine GATA2 expression and dideoxy sequencing to detect KRAS mutation in the same patients in a CRC cohort. We demonstrated that GATA2 was a prospective indicator for poor prognosis in KRAS mutant CRC patients and GATA2-related pathways may be potential new therapeutic targets for KRAS mutant CRC patients.

\section{Materials and methods}

Ethics statement. All procedures performed in studies involving human participants were in accordance with the Ethical Standards of the Research Ethics Committee of Peking University Cancer Hospital and Institute (Beijing, China) (no. 2012071710) and with the 1964 Helsinki Declaration and its later amendments or comparable ethical standards.

Patients and tissue specimens. This retrospective study included a total of 236 patients who were diagnosed with CRC and then received primary tumor resection at the Department of Gastrointestinal Surgery IV, Peking University Cancer Hospital and Institute from 2005 to 2012. All the CRC tissues for immunochemistry analysis were obtained from surgically removed tumors and routinely embedded in paraffin. The clinicopathological characteristics and tumor stages were estimated in accordance with the American Joint Committee on Cancer (AJCC) classification guidelines. Postoperative follow-up results were available for all the 236 patients who have been followed up until March 2015. For the utility of these clinical samples for research, prior written informed consent of the patients and approval from the Research Ethics Committee of Peking University Cancer Hospital and Institute, (Beijing, China) were obtained.

KRAS mutation detection. Formalin-fixed, paraffin-embedded (FFPE) samples with $\geq 50 \%$ tumor cells were collected from patients mentioned above. After extracting genome DNA from the FFPE samples, fragments contained codons 12 and 13 in KRAS exon 2 were amplified by PCR (KRAS forward,
5'-GGTACTGGTGGAGTATTTGATAG-3' and reverse, 5'-TGGTCCTGCACCAGTAATATG-3'). LA Taq polymerase was used to perform the PCR. The product size was $248 \mathrm{bp}$. The reaction mixtures recommended by the manufacturers were used. The initial denaturation step was $5 \mathrm{~min}$ at $94^{\circ} \mathrm{C}$. The thermal profile was 45 cycles of $94^{\circ} \mathrm{C}$ for $30 \mathrm{sec}, 56^{\circ} \mathrm{C}$ for $30 \mathrm{sec}$ and $72^{\circ} \mathrm{C}$ for $20 \mathrm{sec}$. The final elongation was $10 \mathrm{~min}$ at $72^{\circ} \mathrm{C}$. The PCR products were separated on $1 \%$ agarose gel electrophoresis and then sequenced using the same forward primer by Invitrogen 3730 XL genetic analyzer. The sequencing results were analyzed with Chromas software under the condition of signal/noise $>98 \%$ (26).

Immunohistochemistry. Paraffin-embedded tumor tissue blocks were cut 4- $\mu \mathrm{m}$ thick and then baked overnight at $72^{\circ} \mathrm{C}$. Then they were deparaffinized with xylene twice and rehydrated with graded ethanol. The slides were heated in antigen retrieval buffer containing $0.01 \mathrm{M}$ sodium citrate-hydrochloric acid ( $\mathrm{pH}$ 6.0) for $10 \mathrm{~min}$ in a high pressure apparatus at $150^{\circ} \mathrm{C}$. After having been cooled in room temperature for $1 \mathrm{~h}$, the slides were treated with $3 \%$ hydrogen peroxide to inhibit endogenous peroxidase activity. After blocking nonspecific binding with goat serum, the sections were incubated with a rabbit polyclonal antibody against GATA2 (1:200; Santa Cruz Biotechnology) at $4^{\circ} \mathrm{C}$ overnight, followed by incubation with second antibody from the EnVision ${ }^{\mathrm{TM}}$ kit (Dako Cytomation) at room temperature for $30 \mathrm{~min}$. The visualization signal was developed with diaminobenzidine (Sigma). Sections were counterstained with hematoxylin.

Evaluation of GATA2 immunohistochemistry. GATA2 staining scores were determined independently by two pathologists previously uninformed about the histopathological features and patient information to minimize subjectivity. Proportion of positive cells and staining intensity of immunohistochemistry were considered in the scoring process. The scores for GATA2 staining were determined according to the following standard (27): -, no staining or $<10 \%$ positive cells;,$+ 10-20 \%$ weakly to moderately positive cells;,$++ 10-20 \%$ intensively positive cells or $20-50 \%$ weakly positive cells; and +++ , $20-50 \%$ positive cells with moderate to strong reactivity or $>50 \%$ positive cells. All disagreements on results between the two pathologists were resolved after joint review and finally consensus was achieved for all the results.

Statistical analysis. Statistical analysis was carried out using SPSS software 17.0. The Pearson's $\chi^{2}$ verified the correlation between either KRAS gene status or GATA2 expression and clinicopathological parameters. Kaplan-Meier method was used to estimate overall survival curve and differences between groups were compared by the log-rank test. Univariate and multivariate Cox proportional hazard regression models were used to evaluate the predictors for overall survival. A p-value of $<0.05$ was considered to be statistically significant.

\section{Results}

Types of KRAS mutation. Among the 236 enrolled cases, $64(27.1 \%)$ were found to harbor mutations in the KRAS gene. As shown in Table I, the KRAS mutations were 
Table I. Distribution of various KRAS mutant patterns.

\begin{tabular}{lc}
\hline KRAS status & No. of cases $(\%)$ \\
\hline Wild & $172(72.9)$ \\
C12GAT & $31(13.1)$ \\
C13GAC & $12(5.1)$ \\
C12GTT & $12(5.1)$ \\
C12TGT & $5(2.1)$ \\
C12AGT & $2(0.9)$ \\
C12GCT & $1(0.4)$ \\
C13TGC & $1(0.4)$ \\
\hline
\end{tabular}

distributed between codon 12 [51/64 (79.7\%)] and codon 13 [13/64 (20.3\%)]. A total of seven types of mutation in exon 2 were detected. The $\mathrm{G}>\mathrm{A}$ transitions at nucleotides, namely C12GAT and C12AGT in codon 12 as well as C13GAC in codon 13 , were the most frequent mutations in this cohort $(70.3 \%)$.

Association between KRAS mutation or GATA2 expression and clinicopathological features. All 236 patients with CRC were included in this analysis. GATA2 expression was examined in all of the 236 CRC patients (Fig. 1). Table II shows the correlation between either KRAS mutation or GATA2 expression and various clinicopathological features. Statistical analysis indicated that KRAS mutation was significantly correlated with gender $(\mathrm{P}=0.011)$, tumor location $(\mathrm{P}=0.024)$ and overall survival $(\mathrm{P}=0.012)$. Nevertheless, no associations were found between KRAS mutation and age, tumor size, depth of invasion, lymph node metastasis, TNM stage, histological type, and tumor differentiation $(\mathrm{P}>0.05)$. On the other hand, GATA2 expression was significantly linked with age $(\mathrm{P}=0.012)$, depth of invasion $(\mathrm{P}=0.002)$, lymph node metastasis $(\mathrm{P}=0.018)$, TNM stage $(\mathrm{P}=0.001)$ and overall survival $(\mathrm{P}=0.001)$. No correlation was observed between the level of GATA2 expression and gender, tumor location, tumor size, histological type, and tumor differentiation $(\mathrm{P}>0.05)$. However, KRAS mutation was not significantly associated with GATA2 expression $(\mathrm{P}=0.766)$.

Correlation between KRAS mutation or GATA2 expression and overall survival. Kaplan-Meier survival curves with logrank test were performed to evaluate the association between KRAS mutation or GATA2 expression level and prognosis. The results revealed that KRAS mutation in CRC was significantly associated with worse overall survival ( $\mathrm{P}=0.011$, Fig. $2 \mathrm{~A})$. Additionally, GATA2 high expression was significantly correlated with shorter overall survival compared with GATA2 low expression group ( $\mathrm{P}=0.012$, Fig. $2 \mathrm{~B}$ ).

For further analysis, the CRC patients enrolled were separated into different groups according to KRAS status (wild-type and mutant) and GATA2 expression (low and high). The Kaplan-Meier curves in Fig. 2C demonstrated that KRAS mutant/GATA2 high cancers had poor $(\mathrm{P}=0.001$, Fig. 2C) long-term clinical outcomes in comparison with KRAS wild/GATA2 low cancers.
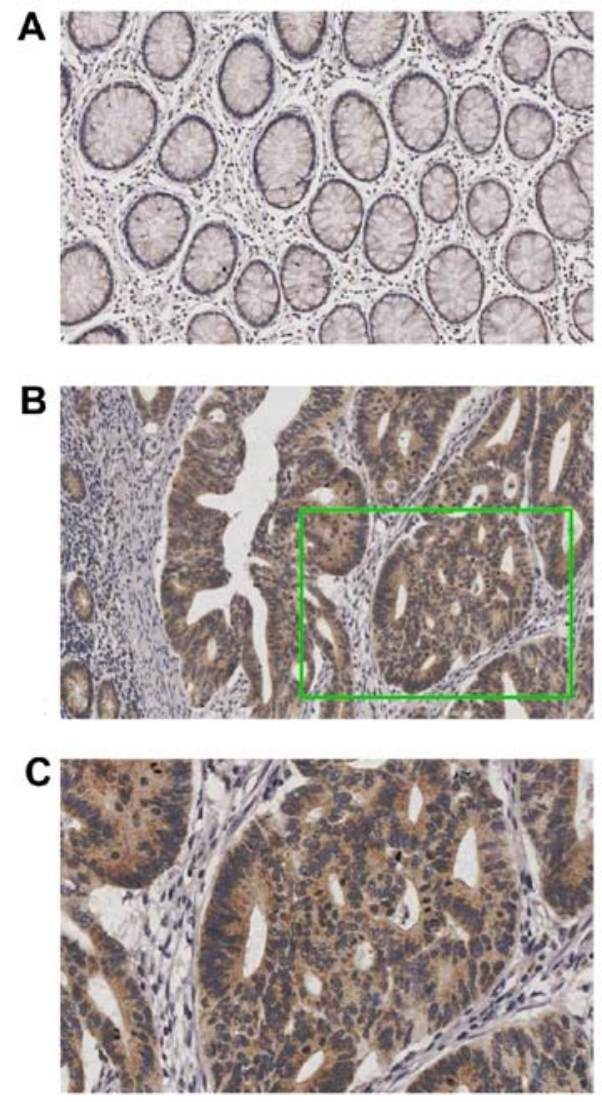

Figure 1. Immunohistochemical results of GATA2 protein expression in normal tissue and colorectal tissue. (A) Representative images revealed negative GATA2 staining in normal tissue, (B) strong GATA2 staining in CRC tissue. Magnification x100. (C) CRC tissue with at magnification $\mathrm{x} 200$.

Stratified analysis by KRAS mutation (KRAS wild-type and KRAS mutant type) showed that in the wild-type KRAS subgroup, no statistical significance was observed between the levels of GATA2 expression and overall survival of CRC patients ( $\mathrm{P}=0.748$, Fig. 3A). However, in the mutant KRAS subgroup, patients with high expression of GATA2 experienced significantly shorter overall survival, compared with those with low expression of GATA2 (P<0.001, Fig. 3B).

Furthermore, the prognostic value of KRAS gene status in different GATA2 protein expression levels were also evaluated. In patients with low GATA2 expression, only a borderline significance was observed in overall survival between KRAS wild-type and KRAS mutant groups ( $\mathrm{P}=0.067$, Fig. 4A). Whereas in patients with high GATA2 expression, mutant KRAS was significantly correlated with poor overall survival, compared with wild-type KRAS ( $\mathrm{P}=0.020$, Fig. 4B).

Cox proportional hazard regression analysis demonstrated GATA2 as an independent prognostic factor. In the univariate analysis, patients with KRAS mutation or GATA-high carcinoma tended to have a shorter overall survival (HR 1.704; 95\% CI 1.123-2.583; $\mathrm{P}=0.012$, HR 1.818; 95\% CI 1.129-2.927; $\mathrm{P}=0.014$, respectively, Table III) than those with KRAS wildgene type or GATA2-low tumors. Gender, tumor location, tumor size, depth of tumor invasion and TNM stage also showed significant associations with overall survival in univariate 
Table II. Correlations between KRAS mutation or GATA2 expression and clinicopathological features in colorectal cancer patients.

\begin{tabular}{|c|c|c|c|c|c|c|c|}
\hline \multirow[b]{2}{*}{ Variables } & \multirow[b]{2}{*}{ Cases } & \multicolumn{2}{|c|}{ KRAS mutation } & \multirow[b]{2}{*}{ P-value } & \multicolumn{2}{|c|}{ GATA2 expression } & \multirow[b]{2}{*}{ P-value } \\
\hline & & Wild-type & Mutant & & Low & High & \\
\hline \multicolumn{8}{|l|}{ Age (years) } \\
\hline$<60$ & 140 & 106 & 34 & 0.237 & 57 & 83 & 0.012 \\
\hline$\geq 60$ & 96 & 66 & 30 & & 24 & 72 & \\
\hline \multicolumn{8}{|l|}{ Gender } \\
\hline Female & 94 & 60 & 34 & 0.011 & 35 & 59 & 0.443 \\
\hline Male & 142 & 112 & 30 & & 46 & 96 & \\
\hline \multicolumn{8}{|l|}{ Tumor location } \\
\hline Colon & 134 & 90 & 44 & 0.024 & 44 & 90 & 0.582 \\
\hline Rectum & 102 & 82 & 20 & & 37 & 65 & \\
\hline \multicolumn{8}{|l|}{ Tumor size $(\mathrm{cm})$} \\
\hline$\leq 4$ & 142 & 108 & 34 & 0.178 & 49 & 93 & 0.941 \\
\hline$>4$ & 94 & 64 & 30 & & 32 & 62 & \\
\hline \multicolumn{8}{|l|}{ Depth of invasion } \\
\hline $\mathrm{T} 1 / \mathrm{T} 2$ & 35 & 30 & 5 & 0.064 & 20 & 15 & 0.002 \\
\hline $\mathrm{T} 3 / \mathrm{T} 4$ & 201 & 142 & 59 & & 61 & 140 & \\
\hline \multicolumn{8}{|c|}{ Lymph node metastasis } \\
\hline Negative & 73 & 53 & 20 & 0.949 & 33 & 40 & 0.018 \\
\hline Positive & 163 & 119 & 44 & & 48 & 115 & \\
\hline \multicolumn{8}{|l|}{ TNM stage } \\
\hline $\mathrm{I} / \mathrm{II}$ & 54 & 39 & 15 & 0.901 & 29 & 25 & 0.001 \\
\hline III/IV & 182 & 133 & 49 & & 52 & 130 & \\
\hline \multicolumn{8}{|l|}{ Histological type } \\
\hline Adenocarcinoma & 213 & 151 & 62 & 0.110 & 74 & 139 & 0.893 \\
\hline Mucinous & 14 & 13 & 1 & & 4 & 10 & \\
\hline Others & 9 & 8 & 1 & & 3 & 6 & \\
\hline \multicolumn{8}{|c|}{ Tumor differentiation } \\
\hline Well & 2 & 1 & 1 & 0.467 & 0 & 2 & 0.259 \\
\hline Moderate & 160 & 116 & 44 & & 50 & 110 & \\
\hline Poor & 53 & 37 & 16 & & 21 & 32 & \\
\hline Unknown & 21 & 18 & 3 & & 10 & 11 & \\
\hline \multicolumn{8}{|l|}{ Survival } \\
\hline Alive & 138 & 109 & 29 & 0.012 & 59 & 79 & 0.001 \\
\hline Dead & 98 & 63 & 35 & & 22 & 76 & \\
\hline
\end{tabular}

analysis (HR 1.639; 95\% CI 1.102-2.437; $\mathrm{P}=0.015$, HR 1.663; 95\% CI 1.097-2.521; $\mathrm{P}=0.017$, HR 1.586; 95\% CI 1.066-2.359; $\mathrm{P}=0.023$, HR 2.575; 95\% CI 1.193-5.559; $\mathrm{P}=0.016$, HR 3.447; 95\% CI 1.832-6.488; P<0.001, respectively, Table III). In the multivariate analysis, the potential risk factors mentioned above were included into the Cox proportional hazard regression model in order to avoid covariation. The results showed that GATA2 high expression, advanced TNM stage (III/IV) and female patients were retained as independent prognostic factors for poor prognosis (HR 1.645; 95\% CI 1.004-2.696; $\mathrm{P}=0.048$, HR 3.058; 95\% CI 1.594-5.865; $\mathrm{P}=0.001$, HR 1.519; 95\% CI 1.010-2.283; $\mathrm{P}=0.045$, Table III).

\section{Discussion}

In this study, we analyzed the influence of KRAS mutation and GATA2 expression on the overall survival based on a cohort of CRC patients. Our data demonstrated that although there was no significant correlation between KRAS mutation and expression of GATA2, high level of GATA2 expression and KRAS mutation were significantly associated with unfavorable prognosis in CRC patients, compared to those bearing GATA2-low and wild-type KRAS tumors, respectively. Moreover, we determined that combined KRAS mutation and overexpression of GATA2 led to more adverse overall survival 

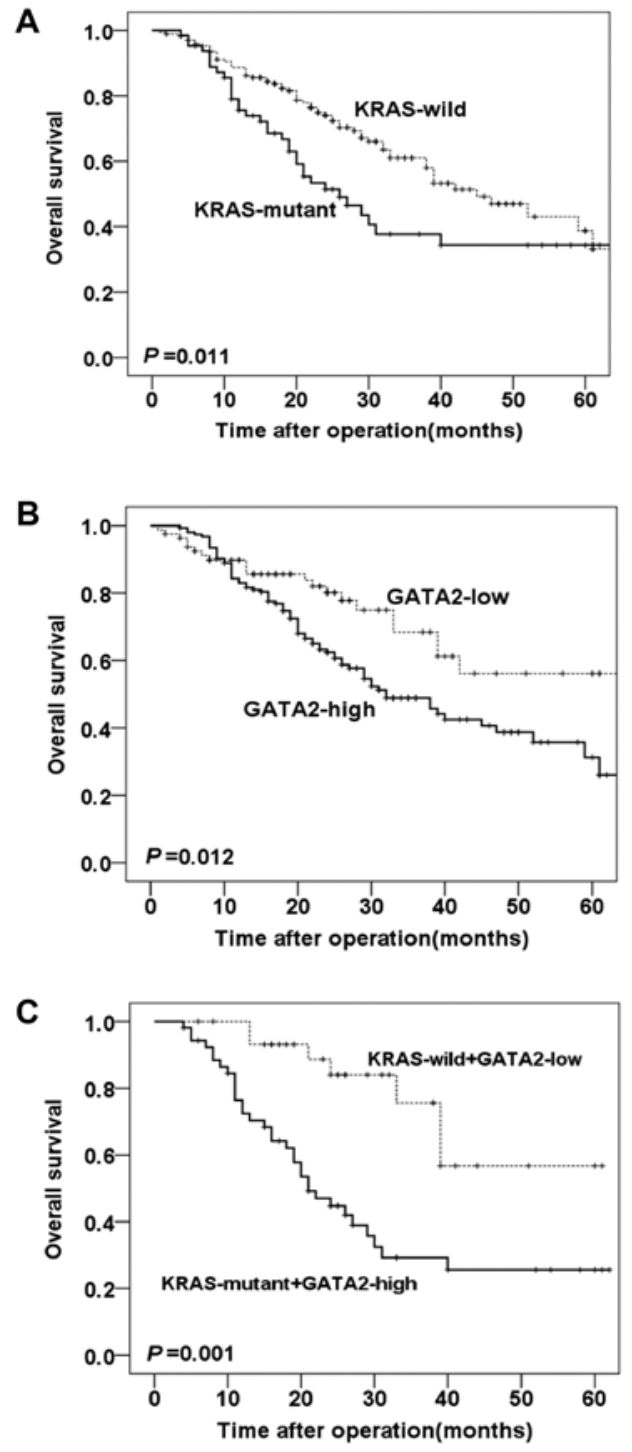

Figure 2. Kaplan-Meier analysis of overall survival in CRC patients. (A) According to KRAS status, (B) according to the levels of GATA2, (C) according to their combination. The log-rank test was used to calculate the p-values.

over the combination of KRAS wild-type and GATA2 low expression. Univariate and multivariate Cox regression analysis demonstrated that the GATA2 expression level was a possible independent predictor for poor overall survival in CRC patients. The results in all showed that high levels of GATA2 expression predicted significant adverse clinical outcomes when KRAS mutation occurred in the same patient, suggesting GATA2 protein may be prone to tumorigenesis of KRAS mutant CRC.

The role of GATA2 in hematopoietic malignant disorders has been well elucidated $(15,19)$. In contrast, only a few studies in gastrointestinal malignancies involving GATA2 transcription factor have been done (28). Previous studies suggested that GATA2 expression had influence on the clinical outcomes in patients with several solid carcinomas, such as breast, prostate, colorectal, renal and hepatocellular carcinoma $(20-23,29,30)$. High levels of GATA2 expression predict tumor recurrence, metastasis, or poor survival except for renal and hepatic cancer $(29,30)$. Moreover, the role of GATA2 in lung cancer
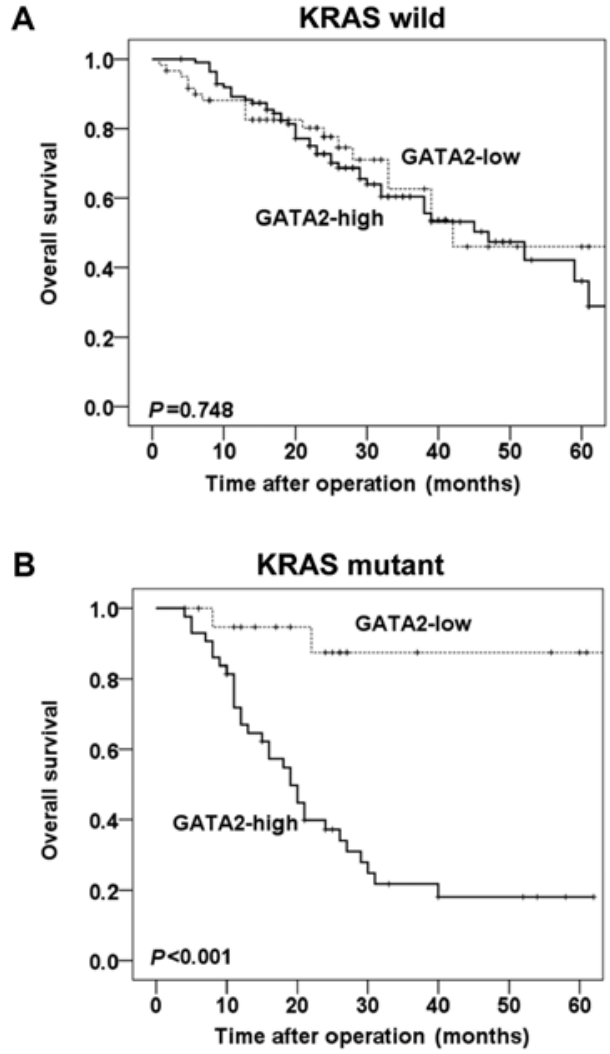

Figure 3. Kaplan-Meier curves for overall survival stratified by KRAS status. (A) KRAS wild-type subgroup according to the expression level of GATA2. (B) KRAS mutant subgroup according to the expression level of GATA2. The log-rank test was used to calculate the p-values.
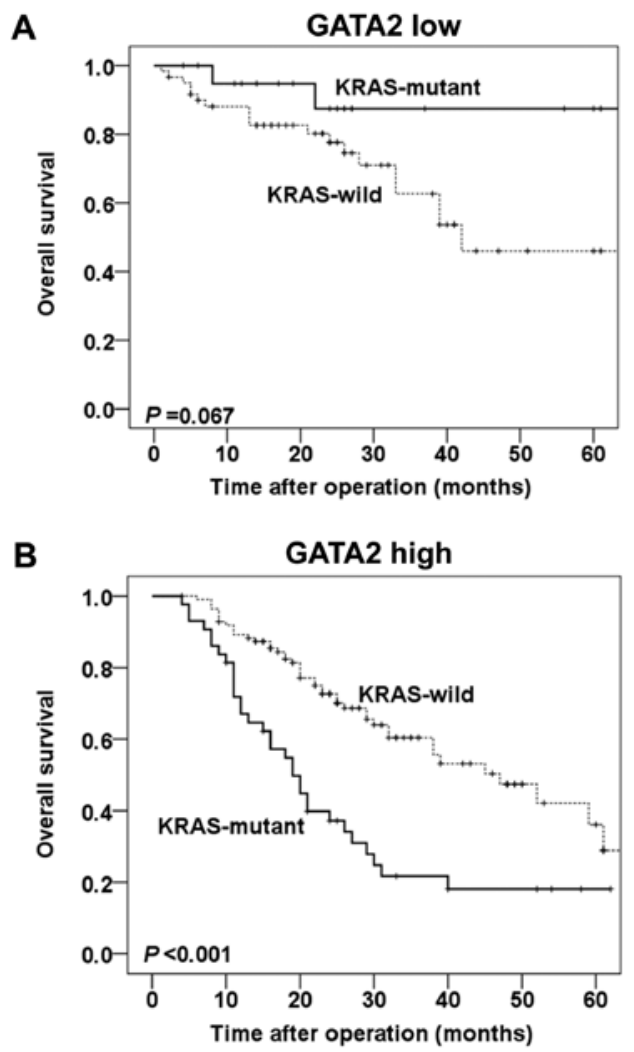

Figure 4. Kaplan-Meier analysis of overall survival stratified by GATA2 expression. (A) Low levels of GATA2 expression subgroup according to KRAS gene status. (B) High levels of GATA2 expression subgroup regarding KRAS gene status. The log-rank test was used to calculate the p-values. 
Table III. Univariate and multivariate analysis of KRAS status and GATA2 expression with respect to overall survival.

\begin{tabular}{|c|c|c|c|c|c|c|}
\hline \multirow[b]{2}{*}{ Variables } & \multicolumn{3}{|c|}{ Univariate } & \multicolumn{3}{|c|}{ Multivariate } \\
\hline & HR & $95 \% \mathrm{CI}$ & P-value & HR & $95 \% \mathrm{CI}$ & P-value \\
\hline Age ( $\geq 60$ vs. $<60$ years) & 1.386 & $0.933-2.060$ & 0.106 & & & \\
\hline Gender (female vs. male) & 1.639 & $1.102-2.437$ & 0.015 & 1.519 & $1.010-2.283$ & 0.045 \\
\hline Tumor location (colon vs. rectum) & 1.663 & $1.097-2.521$ & 0.017 & 1.508 & $0.973-2.335$ & 0.066 \\
\hline Tumor size (>4 vs. $\leq 4 \mathrm{~cm}$ ) & 1.586 & $1.066-2.359$ & 0.023 & 1.368 & $0.902-2.075$ & 0.140 \\
\hline Depth of invasion ( T3/T4 vs. T1/T2) & 2.575 & $1.193-5.559$ & 0.016 & 1.165 & $0.505-2.691$ & 0.720 \\
\hline TNM stage (III/IV vs. I/II) & 3.447 & $1.832-6.488$ & $<0.001$ & 3.058 & $1.594-5.865$ & 0.001 \\
\hline $\begin{array}{l}\text { Histological type } \\
\text { (mucinous vs. adenocarcinoma) }\end{array}$ & 0.578 & $0.183-1.826$ & 0.350 & & & \\
\hline $\begin{array}{l}\text { Tumor differentiation } \\
\text { (poor vs. well/moderate) }\end{array}$ & 1.467 & $0.930-2.315$ & 0.099 & & & \\
\hline KRAS mutation (mutant vs. wild) & 1.704 & $1.123-2.583$ & 0.012 & 1.499 & $0.974-2.305$ & 0.065 \\
\hline GATA2 expression (high vs. low) & 1.818 & $1.129-2.927$ & 0.014 & 1.645 & $1.004-2.696$ & 0.048 \\
\hline
\end{tabular}

remains controversial. A recent study demonstrated that GATA2 levels were indispensable for survival of KRAS mutant NSCLC cells (24). Co-inhibition of GATA2-regulated proteasome and Rho-signaling pathway significantly suppressed the proliferation of lung tumor cells with KRAS mutation. These results indicated that application of novel strategy targeting GATA2 related pathways may benefit patients with RAS pathway mutated NSCLC. On the contrary, Tessema et al reported that GATA2 was not requisite for the survival of lung cancer patients with KRAS mutation due to epigenetic repression (31). Therefore, it is critical to determine the association between KRAS mutation and GATA2 expression, and verify their clinical significance in CRC patients. Consistent with previous studies $(23,24)$, our findings showed that GATA2 played a crucial role in the long-term outcomes of CRC patients with KRAS mutation, which raised the possibility that GATA2 protein may influence carcinogenesis and proliferation of KRAS mutant CRC cells. Accordingly, GATA2 could be a potential target for the treatment of CRC patients with KRAS mutation, which indicates resistance to EGFR-targeting antibodies, including cetuximab and panitumumab. However, GATA2 is traditionally considered to be undruggable (32). Thus, therapies against GATA2-regulated pathways may become an alternative strategy for the treatment of CRC patients with KRAS mutation.

In this cohort, as expected, TNM stage was identified as an independent prognostic factor to predict patient clinical outcomes (HR 3.058; 95\% CI 1.594-5.865; P=0.001, Table III). Additionally, female gender was observed as an adverse factor in our study (HR 1.519; 95\% CI 1.010-2.283; $\mathrm{P}=0.045$, Table III), which was in accordance with previous studies (33).

However, potential limitations still exist in this study. To validate the role of GATA2 expression in CRC patients harboring KRAS mutation, replication cohorts containing detailed clinical data are required. In addition, further functional studies are needed to understand the role of GATA2 and KRAS in CRC.

In conclusion, we demonstrated that elevated GATA2 expression correlated with poor overall survival in CRC patients. Furthermore, GATA2 overexpression in combination with KRAS mutation is significantly associated with poor prognosis. Our results suggested that GATA2 is a promising predictor to identify individuals with worse long-term clinical outcomes, especially in KRAS mutant CRC patients. Also, we consider GATA2-related pathways as potential targets for the development of novel therapies for KRAS mutant CRC.

\section{Acknowledgements}

We thank Dr Bin Dong for histopathological diagnosis. This study was supported by the National Natural Science Foundation of China (no. 81272766 and 81450028), the National High Technology Research and Development Program of China (863 Program, no. 2014AA020603), Beijing Natural Science Foundation (no. 7162039). Beijing Municipal Administration of Hospitals Clinical Medicine Development of Special Funding Support (no. XM201309), Peking University (PKU) 985 Special Funding for Collaborative Research with PKU Hospitals (to X.S. and Fan Bai).

\section{References}

1. DeSantis CE, Lin CC, Mariotto AB, Siegel RL, Stein KD, Kramer JL, Alteri R, Robbins AS and Jemal A: Cancer treatment and survivorship statistics, 2014. CA Cancer J Clin 64: 252-271, 2014.

2. Siegel R, Desantis $C$ and Jemal A: Colorectal cancer statistics, 2014. CA Cancer J Clin 64: 104-117, 2014.

3. Ahmed S, Johnson K, Ahmed $\mathrm{O}$ and Iqbal N: Advances in the management of colorectal cancer: From biology to treatment. Int J Colorectal Dis 29: 1031-1042, 2014.

4. De Rosa M, Pace U, Rega D, Costabile V, Duraturo F, Izzo P and Delrio P: Genetics, diagnosis and management of colorectal cancer (Review). Oncol Rep 34: 1087-1096, 2015.

5. Siegel RL, Miller KD and Jemal A: Cancer statistics, 2015. CA Cancer J Clin 65: 5-29, 2015.

6. Macedo MP, Andrade LB, Coudry R, Crespo R, Gomes M, Lisboa BC, Aguiar S Jr, Soares FA, Carraro DM and Cunha IW: Multiple mutations in the Kras gene in colorectal cancer: Review of the literature with two case reports. Int J Colorectal Dis 26: 1241-1248, 2011.

7. Karapetis CS, Khambata-Ford S, Jonker DJ, O'Callaghan CJ, Tu D, Tebbutt NC, Simes RJ, Chalchal H, Shapiro JD, Robitaille S, et al: K-ras mutations and benefit from cetuximab in advanced colorectal cancer. N Engl J Med 359: 1757-1765, 2008. 
8. Amado RG, Wolf M, Peeters M, Van Cutsem E, Siena S, Freeman DJ, Juan T, Sikorski R, Suggs S, Radinsky R, et al: Wild-type KRAS is required for panitumumab efficacy in patients with metastatic colorectal cancer. J Clin Oncol 26: 1626-1634, 2008.

9. De Roock W, De Vriendt V, Normanno N, Ciardiello F and Tejpar S: KRAS, BRAF, PIK3CA, and PTEN mutations: Implications for targeted therapies in metastatic colorectal cancer. Lancet Oncol 12: 594-603, 2011.

10. Inoue Y, Saigusa S, Iwata T, Okugawa Y, Toiyama Y, Tanaka K, Uchida K, Mohri Y and Kusunoki M: The prognostic value of KRAS mutations in patients with colorectal cancer. Oncol Rep 28: 1579-1584, 2012.

11. Arrington AK, Heinrich EL, Lee W, Duldulao M, Patel S, Sanchez J, Garcia-Aguilar J and Kim J: Prognostic and predictive roles of KRAS mutation in colorectal cancer. Int J Mol Sci 13: 12153-12168, 2012.

12. Kadowaki S, Kakuta M, Takahashi S, Takahashi A, Arai Y, Nishimura Y, Yatsuoka T, Ooki A, Yamaguchi K, Matsuo K, et al: Prognostic value of KRAS and BRAF mutations in curatively resected colorectal cancer. World J Gastroenterol 21 : $1275-1283,2015$.

13. Sameen S, Barbuti R, Milazzo P, Cerone A, Del Re M and Danesi R: Mathematical modeling of drug resistance due to KRAS mutation in colorectal cancer. J Theor Biol 389: 263-273, 2016.

14. Collin M, Dickinson R and Bigley V: Haematopoietic and immune defects associated with GATA2 mutation. $\mathrm{Br}$ J Haematol 169: 173-187, 2015

15. Tsai FY and Orkin SH: Transcription factor GATA-2 is required for proliferation/survival of early hematopoietic cells and mast cell formation, but not for erythroid and myeloid terminal differentiation. Blood 89: 3636-3643, 1997.

16. Ohmori S, Moriguchi T, Noguchi Y, Ikeda M, Kobayashi K, Tomaru N, Ishijima Y, Ohneda O, Yamamoto M and Ohneda $\mathrm{K}$ : GATA2 is critical for the maintenance of cellular identity in differentiated mast cells derived from mouse bone marrow. Blood 125: 3306-3315, 2015.

17. Nandakumar SK, Johnson K, Throm SL, Pestina TI, Neale G and Persons DA: Low-level GATA2 overexpression promotes myeloid progenitor self-renewal and blocks lymphoid differentiation in mice. Exp Hematol 43: 565-577.e1-10, 2015.

18. Hsu AP, McReynolds LJ and Holland SM: GATA2 deficiency. Curr Opin Allergy Clin Immunol 15: 104-109, 2015.

19. Vicente C, Conchillo A, García-Sánchez MA and Odero MD: The role of the GATA2 transcription factor in normal and malignant hematopoiesis. Crit Rev Oncol Hematol 82: 1-17, 2012

20. Wang Y, He X, Ngeow J and Eng C: GATA2 negatively regulates PTEN by preventing nuclear translocation of androgen receptor and by androgen-independent suppression of PTEN transcription in breast cancer. Hum Mol Genet 21: 569-576, 2012.
21. Vidal SJ, Rodriguez-Bravo V, Quinn SA, Rodriguez-Barrueco R, Lujambio A, Williams E, Sun X, de la Iglesia-Vicente J, Lee A, Readhead B, et al: A targetable GATA2-IGF2 axis confers aggressiveness in lethal prostate cancer. Cancer Cell 27: 223-239, 2015.

22. Wu D, Sunkel B, Chen Z, Liu X, Ye Z, Li Q, Grenade C, Ke J, Zhang $\mathrm{C}$, Chen $\mathrm{H}$, et al: Three-tiered role of the pioneer factor GATA2 in promoting androgen-dependent gene expression in prostate cancer. Nucleic Acids Res 42: 3607-3622, 2014.

23. Chen L, Jiang B, Wang Z, Liu M, Ma Y, Yang H, Xing J, Zhang C, Yao Z, Zhang N, et al: Expression and prognostic significance of GATA-binding protein 2 in colorectal cancer. Med Oncol 30: 498, 2013.

24. Kumar MS, Hancock DC, Molina-Arcas M, Steckel M, East P, Diefenbacher M, Armenteros-Monterroso E, Lassailly F, Matthews N, Nye E, et al: The GATA2 transcriptional network is requisite for RAS oncogene-driven non-small cell lung cancer. Cell 149: 642-655, 2012

25. Shen S, Mao CQ, Yang XZ, Du XJ, Liu Y, Zhu YH and Wang J: Cationic lipid-assisted polymeric nanoparticle mediated GATA2 siRNA delivery for synthetic lethal therapy of KRAS mutant non-small-cell lung carcinoma. Mol Pharm 11: 2612-2622, 2014.

26. Gao J, Li YY, Sun PN and Shen L: Comparative analysis of dideoxy sequencing, the KRAS StripAssay and pyrosequencing for detection of KRAS mutation. World J Gastroenterol 16 : 4858-4864, 2010

27. Xing X, Peng L, Qu L, Ren T, Dong B, Su X and Shou C: Prognostic value of PRL-3 overexpression in early stages of colonic cancer. Histopathology 54: 309-318, 2009.

28. Ayanbule F, Belaguli NS and Berger DH: GATA factors in gastrointestinal malignancy. World J Surg 35: 1757-1765, 2011.

29. Peters I, Dubrowinskaja N, Tezval H, Kramer MW, von Klot CA, Hennenlotter J, Stenzl A, Scherer R, Kuczyk MA and Serth J: Decreased mRNA expression of GATA1 and GATA2 is associated with tumor aggressiveness and poor outcome in clear cell renal cell carcinoma. Target Oncol 10: 267-275, 2015.

30. Li YW, Wang JX, Yin X, Qiu SJ, Wu H, Liao R, Yi Y, Xiao YS, Zhou J, Zhang BH, et al: Decreased expression of GATA2 promoted proliferation, migration and invasion of HepG2 in vitro and correlated with poor prognosis of hepatocellular carcinoma. PLoS One 9: e87505, 2014.

31. Tessema M, Yingling CM, Snider AM, Do K, Juri DE, Picchi MA, Zhang X, Liu Y, Leng S, Tellez CS, et al: GATA2 is epigenetically repressed in human and mouse lung tumors and is not requisite for survival of KRAS mutant lung cancer. J Thorac Oncol 9: 784-793, 2014.

32. Darnell JE Jr: Transcription factors as targets for cancer therapy. Nat Rev Cancer 2: 740-749, 2002.

33. Kim SE, Paik HY, Yoon H, Lee JE, Kim N and Sung MK: Sex- and gender-specific disparities in colorectal cancer risk. World J Gastroenterol 21: 5167-5175, 2015. 